\title{
Extracting Respiratory Motion from 4D MRI using Organ-wise Registration
}

\author{
Ashrani Aizzuddin Abd. Rahnib ${ }^{b, a}$, Rhodri Smith $^{a}$, Emma Lewis $^{a}$ and Kevin Wells ${ }^{a}$ \\ ${ }^{a}$ Centre for Vision, Speech and Signal Processing, University of Surrey, Guildford, GU2 7XH; \\ ${ }^{b}$ Universiti Kebangsaan Malaysia, Malaysia
}

\begin{abstract}
Nuclear Medicine (NM) imaging serves as a powerful diagnostic tool for imaging of biochemical and physiological processes in vivo. The degradation in spatial image resolution caused by the often irregular respiratory motion must be corrected to achieve high resolution imaging. In order perform motion correction more accurately, it is proposed that patient motion obtained from 4D MRI can be used to analyse respiratory motion. To extract motion from the dynamic MRI dataset an organ wise intensity based affine registration framework is proposed and evaluated. Comparison of the resultant motion obtained within selected organs is made against an open source free form deformation algorithm. For validation, the correlation of the results of both techniques to a previous study of motion in 20 patients is found. Organwise affine registration correlates very well $(r \approx 0.9)$ with a previous study (Segars et al., 2007) ${ }^{1}$ whilst free form deformation shows little correlation $(r \approx 0.3)$. This increases the confidence of the organ wise affine registration framework being an effective tool to extract motion from dynamic anatomical datasets.
\end{abstract}

Keywords: Respiratory motion, Segmentation, Registration, 4D MRI

\section{INTRODUCTION}

Respiratory motion degrades quantitatively and qualitatively the functional information obtained from nuclear medicine images. To improve disagnostic confidence and accuracy this motion must be corrected. Popular approaches sum individual frames obtained from respiratory gating. ${ }^{2}$ Gating is reliant on the patient maintaining an average consistent respiratory cycle throughout the investigation which is frequently unrealistic. ${ }^{3}$ A flexible framework has previously been investigated which proposes to derive an estimate of the motion throughout the nuclear medicine study from a 4D anatomical training dataset. This allows for the correction of irregular, unseen respiratory patterns. ${ }^{4}$ This paper describes the method of extracting such modal data of respiratory motion via temporal adaptation based on a 4D MRI dataset (Fig. 1). MRI is chosen as it provides no radiation burden to the patient with good soft tissue contrast in comparison to CT. 4D MRI falls into the category of time resolved (continuous) volumetric imaging; ${ }^{5}$ thus representing "free" breathing. A semi automated organ segmentation approach, followed by intensity based organ wise affine registration is presented. Processing for motion is performed by organ to account for the relative motion between organs; ${ }^{6}$

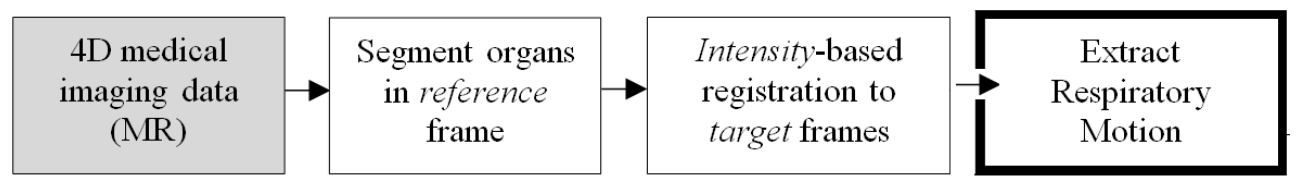

Figure 1. Diagram illustrating the process of obtaining respiratory motion from 4D dataset (shaded box).

\section{METHODS}

MRI data were acquired using the Siemens MAGNETOM®Verio 3T MRI scanner. Two types of volumetric images were acquired, an anatomical image volume and dynamic image volumes. The anatomical image volume have an in-slice resolution of $0.977 \mathrm{~mm}$ and a slice separation of $10.5 \mathrm{~mm}$. Slices were acquired in all three orthogonal directions (axial, coronal and sagittal). The dynamic volumes have an in-plane resolution of 1.534

Medical Imaging 2013: Image Processing, edited by Sebastien Ourselin, David R. Haynor, Proc. of SPIE Vol. 8669, 866930 - C 2013 SPIE - CCC code: 1605-7422/13/\$18

doi: $10.1117 / 12.2006861$

Proc. of SPIE Vol. $8669866930-1$ 
$\mathrm{mm}$ and slice separation of $5 \mathrm{~mm}$. Only coronal slices were acquired for the dynamic volumes. The volumes were acquired every $0.956 \mathrm{~s}$ in two sets of sequences of 10 volumes each, which were acquired tens of minutes apart from one another.

\subsection{Segmentation of Organs}

Segmentation of individual organs was achieved using active contours ${ }^{7}$ whereby a completed contour for one slice becomes the approximate contour for the next slice. Firstly contours in the orthogonal anatomical slices are found and then used as a guideline for contouring in the first volume of the dynamic sequence, which is designated as the reference volume for the entire sequence. Some minor manual refinement can be performed if necessary. The organs are only segmented in the reference frame of the dynamic sequence. This segmentation is then used as a template for intensity based registration to other frames in the $4 \mathrm{D}$ imaging sequence. This ensures that any inaccuracy in the $4 \mathrm{D}$ respiratory motion obtained is from registration and not inconsistencies in segmentation. A rendering of the segmented anatomy is shown in Fig. 2; the organs chosen for registration are the lungs, heart, liver, stomach, spleen and kidneys.

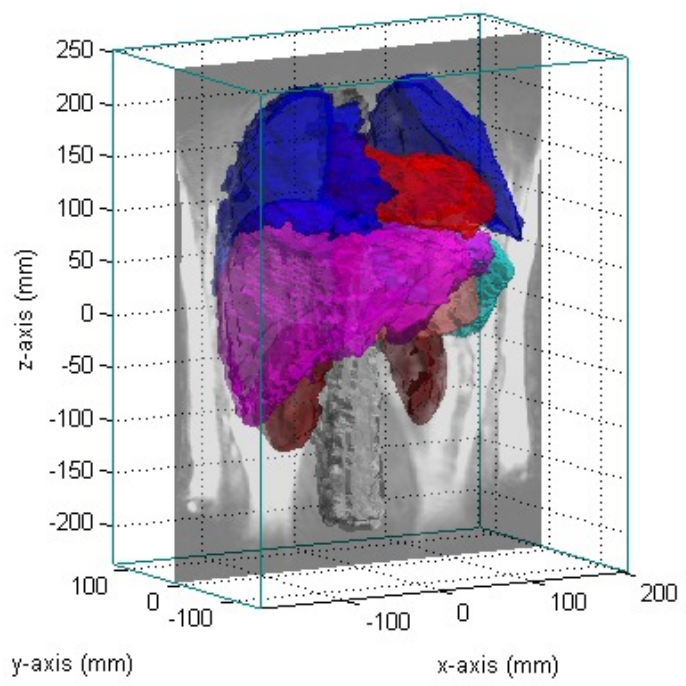

Figure 2. A rendering of the segmented anatomy together with a slice from the first volume of the first dynamic MRI sequence. The bounding box shows the extent of the volume. The rendered organs are the lungs (blue), heart (red), liver (magenta), stomach (peach), spleen (cyan), kidneys (dark red) and spine (grey).

\subsection{Registration of Organs}

In this study organ deformations are assumed to be affine as in (Dey et al., 2009). ${ }^{8}$ The registration is performed in two phases; firstly a rigid deformation is found which is used to initialise the solution of an affine deformation. The transformations for registration are found using the intensity values of the reference image at the locations of the underlying segmented organs. The reference image $\mathcal{A}$ is then transformed $\left(\mathcal{A}^{\prime}\right)$ to the target frames $\mathcal{B}$ within the dynamic sequence. The transformation chosen maximizes the similarity between the two images by minimizing the sum of square differences (SSD) of intensities between voxels (equation 1). ${ }^{9}$

$$
S_{S S D}\left(\mathcal{A}^{\prime}, \mathcal{B}\right)=\sum_{i=1}^{N}\left(\mathcal{A}(i)^{\prime}-\mathcal{B}(i)\right)^{2},
$$

This similarity measure is optimal when the dissimilarity between frames are assumed to have a Gaussian distribution. ${ }^{9}$

Additionally, an extended ROI is defined for each organ so as to include its boundary in registration. This ROI is defined by isotropic dilation of the segmented organ. The amount of dilation used is $1 \mathrm{~cm}$ around the segmented boundary. Fig 3 shows this ROI for the liver. 


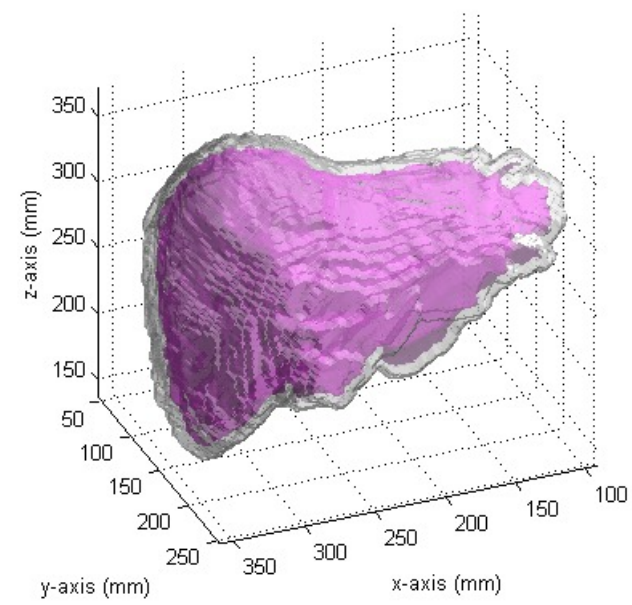

Figure 3. Render of the liver (magenta) with the extended ROI (white).

\section{RESULTS AND DISCUSSION}

To evaluate the effectiveness of the proposed method the mean trajectory of all voxels in the chosen organs were analysed. A comparison was made against a free form deformation (FFD) based approach, over the whole image volume, using the NiftyReg registration package. ${ }^{10,11}$ The default NiftyReg parameter values were used in the evaluation.

Figs. 4(a) and (b) shows the mean value of maximum displacement in the $\mathrm{x}-, \mathrm{y}$ - and $\mathrm{z}$-axes over all voxels within the chosen organs. Figs. 4(c) shows the mean value of maximum 3D Euclidean translation over the first 4D sequence, averaged over all voxels. Figs. 4(d) shows the mean values over all voxels in all chosen organs. The error bars indicate the standard deviation over all voxels. As can be seen FFD estimates more motion than organ-wise affine registration. Noticeably a larger motion is estimated in the y-axis (AP direction) than the amplitude in the $\mathrm{z}$ axis (SI direction).

Figs. 5)(a) to (d) show the same analysis as Figs. 4(a) to (d) for the second sequence. Here FFD estimates slightly less motion than organ-wise affine registration. However, as in Fig. 4, a larger motion is estimated in the $\mathrm{y}$-axis (AP direction) than the amplitude in the $\mathrm{z}$ axis (SI direction).

The detected motion from both techniques in both sequences (Figs. 4 and. 5) is compared to a previous study ${ }^{1}$ whereby organ motion was found via manual segmentation in 20 patients. Fig. 6 shows the mean of detected motion from this study. Error bars representing the standard deviation over all organs was not available in Fig. 6. Separate values for each lung are also not available. The $\mathrm{z}$ axis (SI) motion of the lungs was not stated and is thus considered to be the mean z-axis (SI) motion of organs below the diaphragm (liver, stomach and spleen).

The correlation between the results found by (Segars et al., 2007) ${ }^{1}$ and that found by organ wise affine registration and FFD was evaluated. The correlation is quantified over the proportion of motion between the x-, y- and z-axes. This measure of correlation for both sequences is shown in Fig. 7.

The average correlation over all organs for the proportion of motion between the $\mathrm{x}-, \mathrm{y}-\mathrm{and} \mathrm{z}$-axes for the both sequences was 0.9 for the affine technique whilst below 0.3 for the FFD method. The proposed approach also agrees with previous measurements of respiratory motion which have noted larger amounts of motion in the SI direction ${ }^{12}$ rather than the AP, more fitting with the estimates obtained from organ-wise affine registration.

\section{CONCLUSION}

The results here suggests that affine organ-wise registration is a reasonable method for extraction of respiratory motion from 4D MRI. The motion found have a proportion of motion in the $\mathrm{x}-, \mathrm{y}$ - and z-axes that are as expected from previous studies. ${ }^{1,12}$ The motion outside of the chosen organs was not considered here. However this can 


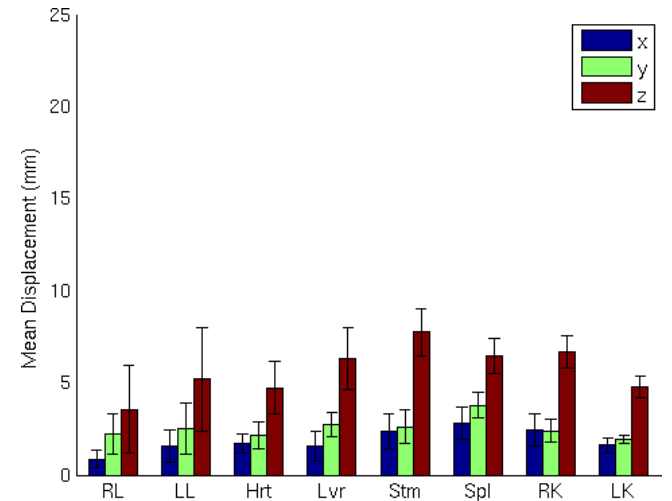

(a) Maximum component displacement for organ-wise affine registration.

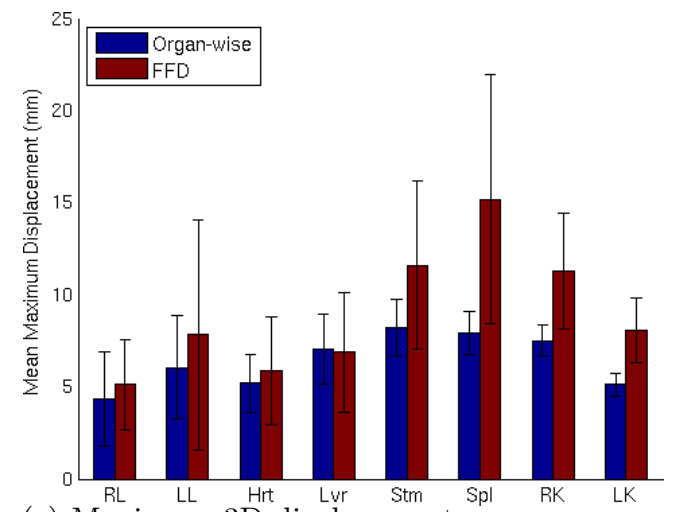

(c) Maximum 3D displacement over sequence.

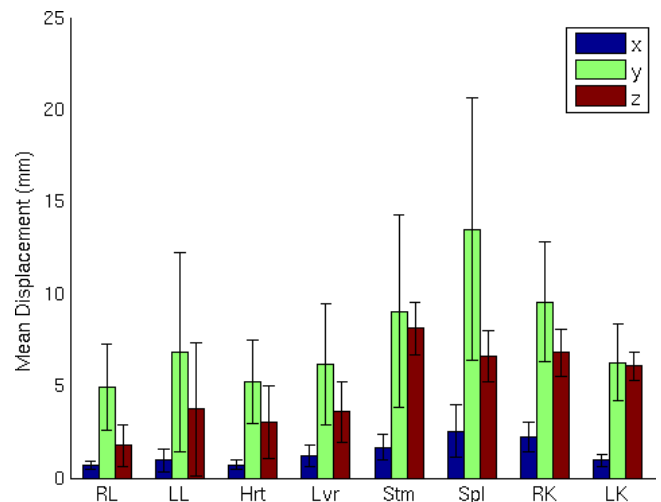

(b) Maximum component displacement for FFD registration.

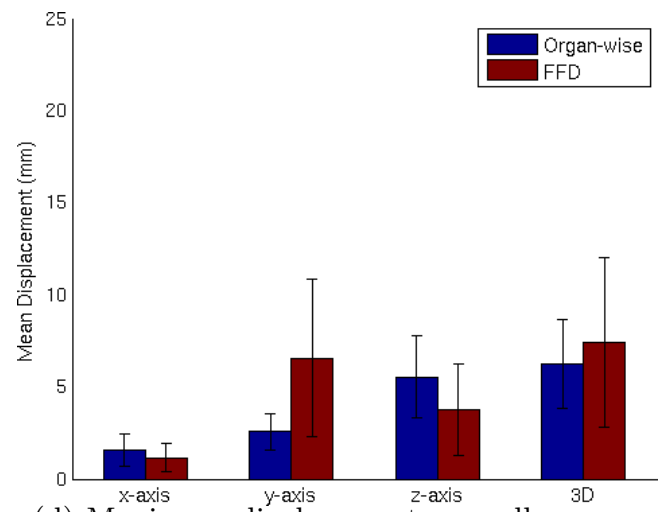

(d) Maximum displacement over all organs.

Figure 4. Analysis of the mean trajectory of all voxels in the chosen organs for the first sequence. The labels of the chosen organs are: RL: right lung, LL: left lung, Hrt: heart, Lvr: liver, Stm: Stomach, Spl: spleen, RK: right kidney and LK: left kidney.

be performed by the scheme described in (Jones et al., 2009). ${ }^{6}$ With this extension, the motion field found from affine organ-wise registration can then be used as an initial deformation for an FFD method for greater accuracy.

\section{Acknowledgement}

Ashrani Aizzuddin Abd. Rahni was funded by the Malaysian Ministry of Higher Education and University Kebangsaan Malaysia. Rhodri Smith is funded by the UK EPSRC.

\section{REFERENCES}

[1] W. P. Segars, S. Mori, G. T. Y. Chen, and B. M. W. Tsui, "Modeling respiratory motion variations in the 4D NCAT phantom," in IEEE NSS/MIC Conf Rec, 4, pp. 2677-2679, Nov. 2007.

[2] M. Dawood and F. Büther and L. Stegger and X. Jiang and K. P. Schäfers, "Respiratory Motion Correction in 3D PET Data with Advanced Optical Flow Algorithms," IEEE Trans Med Imag 27, pp. 1164-1175, Jul 2008.

[3] A. King, C. Buerger, C. Tsoumpas, P. Marsden, and T. Schaeffter, "Thoracic respiratory motion estimation from MRI using a statistical model and a 2-D image navigator," Med. Image Anal. 16(1), pp. 252-264, 2012.

[4] A. A. Abd. Rahni, E. Lewis, M. J. Guy, B. Goswami, and K. Wells, "A Particle Filter Approach to Respiratory Motion Estimation in Nuclear Medicine Imaging," IEEE Trans Nuc Sci 58, pp. 2276-2285, Oct 2011. 


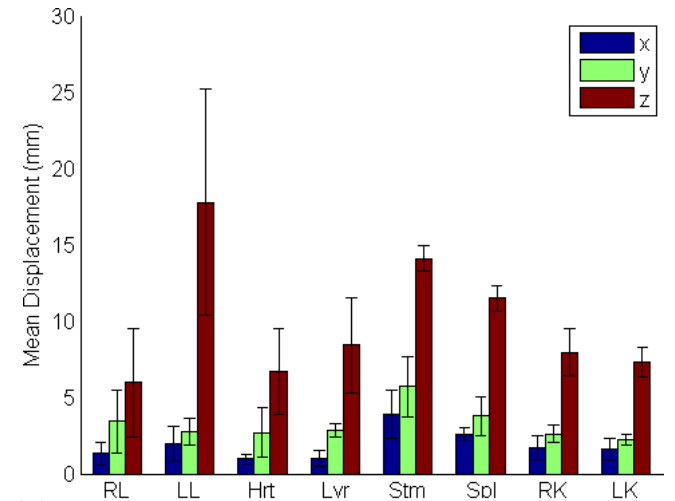

(a) Maximum component displacement for organ-wise affine registration.

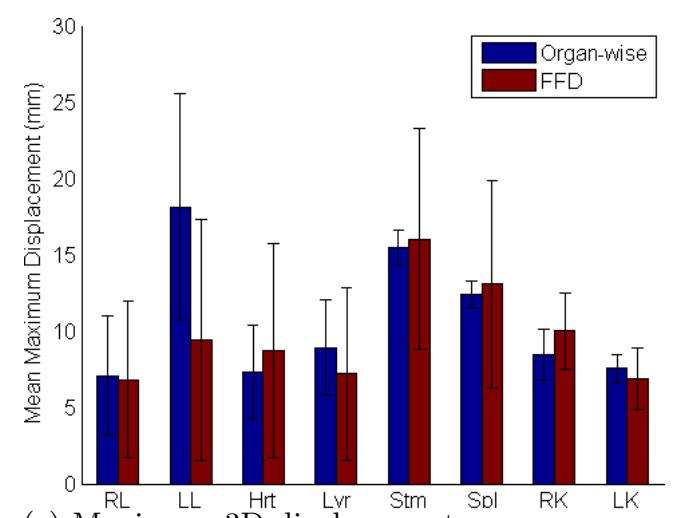

(c) Maximum 3D displacement over sequence.

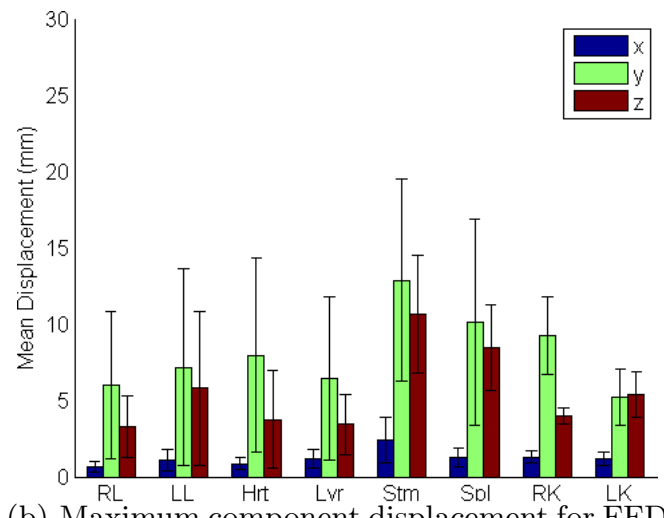

(b) Maximum component displacement for FFD registration.

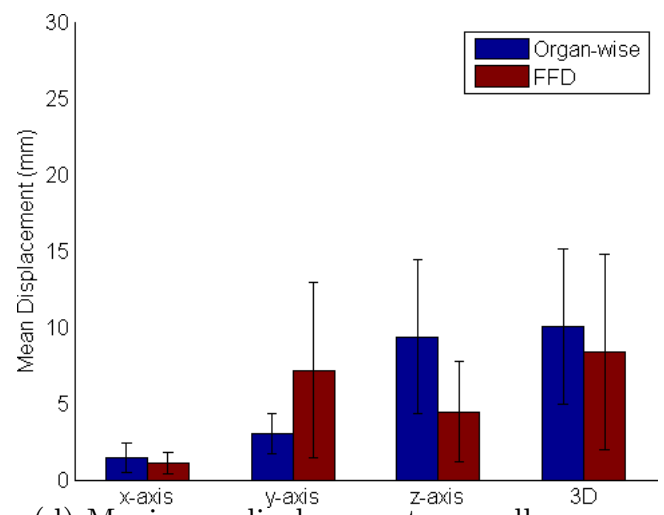

(d) Maximum displacement over all organs.

Figure 5. Analysis of the mean trajectory of all voxels in the chosen organs for the second sequence. The labels of the chosen organs are: RL: right lung, LL: left lung, Hrt: heart, Lvr: liver, Stm: Stomach, Spl: spleen, RK: right kidney and LK: left kidney.

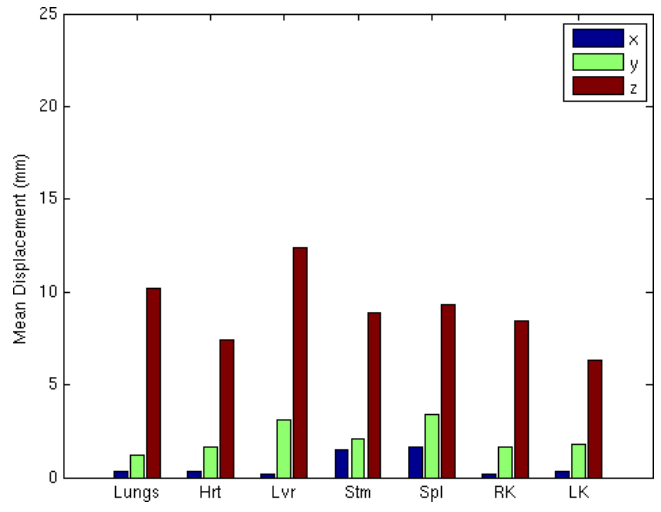

Figure 6. Mean displacement from a previous study. ${ }^{1}$ The labels for the other organs with the exception of the lungs are the same as Fig. 4.

[5] J. Biederer, C. Hintze, M. Fabel, and J. Dinkel, "Magnetic resonance imaging and computed tomography of respiratory mechanics," J Magn Reson Imaging 32(6), pp. 1388-1397, 2010.

[6] J. Jones, E. Lewis, M. Guy, and K. Wells, "A virtual dissection based registration to model patient-specific respiratory motion," in IEEE NSS/MIC Conf Rec, pp. 3571-3576, 2009.

[7] S. Lankton, "Sparse Field Methods," tech. rep., Georgia Institute of Technology, Apr. 2009. 


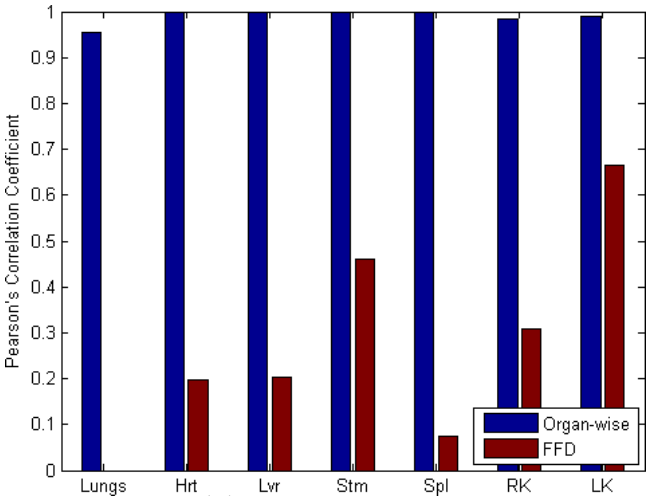

(a) First sequence

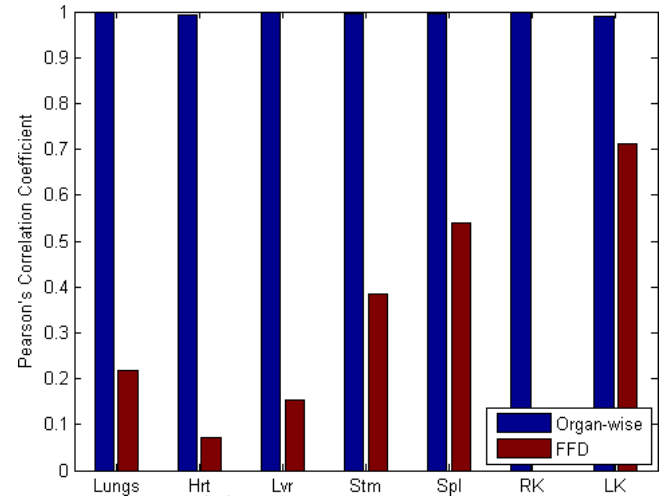

(b) Second sequence

Figure 7. Correlation of component displacements.

[8] J. Dey et al., "Estimation of Cardiac Respiratory-Motion by Semi-Automatic Segmentation and Registration of Non-Contrast-Enhanced 4D-CT Cardiac Datasets," IEEE Trans Nuc Sci 56, pp. 3662-3671, Dec 2009.

[9] M. Sonka and J. M. Fitzpatrick, eds., Handbook of Medical Imaging: Medical Image Processing and Analysis, SPIE, 2009.

[10] M. Modat, G. R. Ridgway, Z. A. Taylor, M. Lehmann, J. Barnes, D. J. Hawkes, N. C. Fox, and S. Ourselin, "Fast free-form deformation using graphics processing units," Comput. Methods Programs Biomed. 98(3), pp. 278-284, 2010.

[11] S. Ourselin, R. Stefanescu, and X. Pennec, "Robust Registration of Multi-modal Images: Towards RealTime Clinical Applications," in MICCAI, 2489, pp. 140-147, 2002.

[12] P. J. Keall, G. S. Mageras, J. M. Balter, R. S. Emery, K. M. Forster, S. B. Jiang, J. M. Kapatoes, D. A. Low, M. J. Murphy, B. R. Murray, C. R. Ramsey, M. B. van Herk, S. S. Vedam, J. W. Wong, and E. Yorke, "The management of respiratory motion in radiation oncology report of AAPM Task Group 76," tech. rep., American Association of Physicists in Medicine, 2006. 